\title{
Prediction of the surgical outcome for the treatment of cervical myelopathy by using hyperbaric oxygen therapy
}

\author{
H Ishihara, H Matsui, H Kitagawa, T Yonezawa and H Tsuji \\ Department of Orthopaedic Surgery, Toyama Medical and Pharmaceutical University, Toyama, Japan
}

\begin{abstract}
The effectiveness of hyperbaric oxygen therapy (HBO) in predicting the recovery after surgery in patients with cervical compression myelopathy was evaluated. HBO has been used to treat brain and spinal cord diseases, but the effect is generally temporary. This is the first paper to utilize $\mathrm{HBO}$ as a diagnostic tool to evaluate the functional integrity of the spinal cord. The study group consisted of 41 cervical myelopathy patients aged $32-78$ years. Before surgery, the effect of HBO was evaluated and was categorized as four grades. The severity of the myelopathy and the recovery after surgery were evaluated by the score proposed by the Japanese Orthopaedic Association (JOA score). The correlation between many clinical parameters including the HBO effect and the recovery rate of JOA score was evaluated. The recovery rate of JOA score was found to be $75.2 \pm 20.8 \%$ in the excellent group, $78.1 \pm 17.0 \%$ in the good group, $66.7 \pm 21.9 \%$ in the fair group and $31.7 \pm 16.4 \%$ in the poor group. There was a statistically significant correlation between the $\mathrm{HBO}$ effect and the recovery rate of the JOA score after surgery $(r=0.641, P<0.0001)$. The effect of $\mathrm{HBO}$ showed a high correlation with the recovery rate after surgery as compared to the other investigated parameters. HBO can be employed to assess the chance of recovery of spinal cord function after surgical decompression.
\end{abstract}

Keywords: cervical myelopathy; hyperbaric oxygen therapy; surgical outcome

\section{Introduction}

Although the surgical results of cervical compression myelopathy are relatively good by virtue of improvement of the surgical procedures, there are some cases where the results are unfavorable, probably due to an irreversible change of the spinal cord. ${ }^{5,6}$ It is clinically important to predict the results of surgical decompression so that one can inform patients about the likely extent of neurological recovery after surgery. Hyperbaric oxygen therapy (HBO) has been used to treat cerebral-spinal cord diseases, ${ }^{4}$ but the effect is generally temporary. We have attempted to utilize $\mathrm{HBO}$ as a prognostic tool to evaluate the functional integrity of the spinal cord. In the present study we assessed the usefulness of $\mathrm{HBO}$ to predict the effect of surgical decompression in patients with cervical compression myelopathy.

\section{Patients and methods}

The study group consisted of 41 cervical myelopathy patients (27 men and 14 women) aged $32-78$ years (average age: 60 years) who underwent surgery at the university hospital. The average length of follow-up was 2 years (range: 5 months to 4.4 years). Eighteen

Correspondence: Hirokazu Ishihara, MD patients with cervical spondylotic myelopathy (CSM), 17 with ossification of the posterior longitudinal ligament (OPLL) and six with intervertebral disc herniation (IDH) were included. Six patients underwent anterior interbody fusion using Simmons' method, ${ }^{17} 33$ patients underwent expanisve laminoplasty $^{6}$ and two patients underwent both anterior interbody fusion and laminoplasty. Before surgery, the effect of $\mathrm{HBO}$ was evaluated. HBO was performed at 2.5 atmospheric pressure under $100 \%$ oxygen for one hour in a specially equipped chamber (RHS3/OC, Vickers, Hampshire, UK). The HBO effect was assessed by the improvement of subjective symptoms such as numbness or clumsiness of the hands. The $\mathrm{HBO}$ effect was categorized in four grades according to the duration of symptom improvement regardless of the extent of improvement: excellent (the HBO effect continued over $24 \mathrm{~h}$ ), good (continuing for $24 \mathrm{~h}$ ), fair (the effect appeared only when the patient was in the HBO chamber) and poor (no response). The severity of the myelopathy was evaluated before surgery, and at the final follow-up by the functional assessment score proposed by the Japanese Orthopaedic Association (JOA score) (Table 1). The recovery rate after surgery was evaluated using the formula shown in Table 1.5 The correlation between the $\mathrm{HBO}$ effect and the recovery rate of the JOA score was evaluated. To 
minimize the observer's preconception, the JOA score was assessed by doctors who did not know the result of the $\mathrm{HBO}$ effect. We also evaluated the correlation between the $\mathrm{HBO}$ effect and the following parameters: age at surgery, gender, original disease (CSM, OPLL, IDH), level and type of myelopathy (Crandall's classification: ${ }^{1}$ transverse lesion syndrome, motor system syndrome, central cord syndrome, BrownSéquard syndrome and brachialgia and cord syndrome), duration of myelopathy, surgical method (anterior interbody fusion, laminoplasty, combined surgery), the grip-and-release test ${ }^{15}$ (the patient is asked to grip and release his fingers as rapidly as possible. The number of complete cycles of finger movement within 10 seconds is counted; a normal result is 20 or more), anteroposterior spinal canal diameter at the most severe compression level in plain lateral radiographs, the compression ratio (diameter of the dural sac at the most severe compression level/ diameter of the dural sac at the normal level) in myelography, the transverse area of the spinal cord at the most severe compression level in computed tomography after myelography (CTM) measured by an image analyzer system (CIA-102, Olympus, Tokyo, Japan), and the presence of an intramedullary high intensity region in T2-weighted saggital magnetic resonance imaging $(\mathrm{MRI})$.

\section{Statistical analysis}

The results were expressed as means \pm standard deviation. We selected the statistically significant parameters for prediction of the recovery rate of JOA score by Mann-Whitney's U test, Kruskal-Wallis rank test with Fisher's protected least significant difference (Fisher's PLSD) and Spearman's rank correlation. A $P$ value less than 0.05 was considered significant. Using these statistically significant parameters for the prediction of the recovery rate of the JOA score, we obtained the recovery rate estimating formula by employing the multiple regression method.

\section{Results}

The results were excellent in 15 patients, good in nine, fair in five, and poor in 12. The results of the clinical parameters of each group are shown in Table 2.

\section{Relationship between the $\mathrm{HBO}$ effect and the recovery rate of $\mathrm{JOA}$ score}

The recovery rate of JOA score after surgery was found to be $75.2 \pm 20.8 \%$ in the excellent group, $78.1 \pm 17.0 \%$ in the good group, $66.7 \pm 21.9 \%$ in the fair group and $31.7 \pm 16.4 \%$ in the poor group. The recovery rate of JOA score in the poor group was significantly worse than that of other groups $(P<0.0001$ to the excellent group, $P<0.0001$ to the good group, $P=0.0021$ to fair group. Kruskal-Wallis rank test with Fisher's PLSD). But there was no statistically significant difference
Table 1 Criteria of evaluation of the operative results of patients with cervical myelopathy by the Japanese Orthopaedic Association (JOA score) and the formula of recovery rate after surgery ${ }^{5}$

I. Upper extremity function

0 . Impossible to eat with either chopsticks or spoon

1. Possible to eat with spoon, but not with chopsticks

2. Possible to eat with chopsticks, but inadequate

3. Possible to eat with chopsticks, but awkward

4. Normal

II. Lower extremity function

0 . Impossible to walk

1. Needs cane or aid on flat ground

2. Needs cane or aid only on stairs

3. Possible to walk without cane or aid, but slow

4. Normal

III. Sensory

A. Upper extremity

0. Apparent sensory loss

1. Minimal sensory loss

2. Normal

B. Lower extremity The same as A

C. Trunk

The same as A

IV. Bladder function

0. Complete retention

1. Severe disturbance

(Inadequate evaluation of the bladder, straining, dribbling urine)

2. Mild disturbance

(Urinary frequency, urinary hesitancy)

3. Normal

Recovery rate $=[$ Postoperative JOA score (final follow-up) - Preoperative JOA score 17 (full score) - Preoperative JOA score] $\times 100(\%)$

among the excellent, good and fair groups. Spearman's rank correlation coefficient between the grades of the HBO effect and the recovery rate of JOA score after surgery was $0.641 \quad(P<0.0001)$ (Table 3$)$. From the comparison of the clinical parameters between the poor group and the other groups, the rate of positive intramedullary high intensity region in T2-weighted MRI in the poor group was significantly higher than that of the excellent, good and fair groups (Table 4).

\section{Relationship between clinical parameters and the recovery rate of $J O A$ score}

The result of the grip-and-release test was also a statistically significant parameter to correlate with the recovery rate of JOA score after surgery. The correlation coefficient was $0.381 \quad(P=0.0184)$ (Table $3)$. But the other parameters did not correlate with the recovery rate of JOA score after surgery. Using the HBO effect and the result of the grip-and-release test, the recovery rate of the JOA score after surgery was estimated by the following formula: the recovery rate of JOA score $(\%)=24.67+42.01 \times \mathrm{HBO}$ effect (the excellent, good and fair groups $=1$, the poor group $=0$ ) 


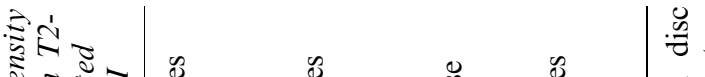

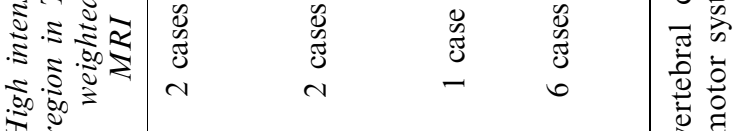

$\Xi:$

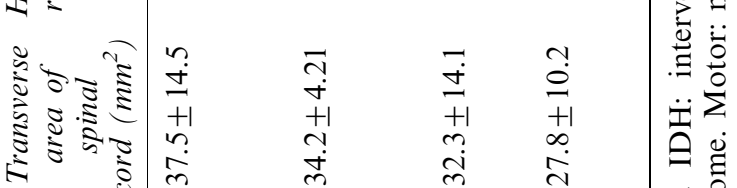

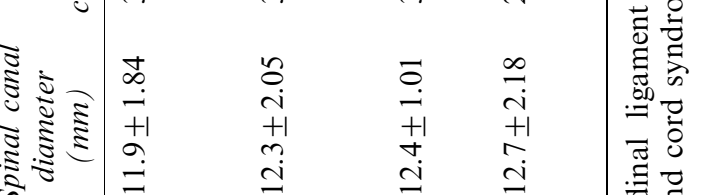

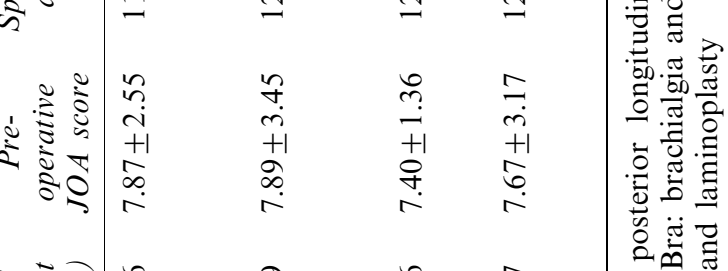

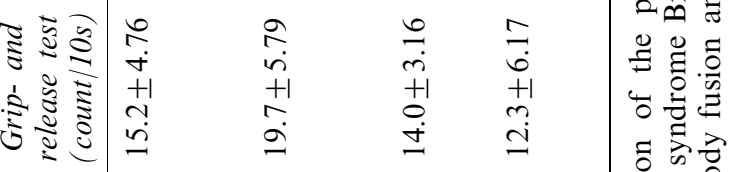

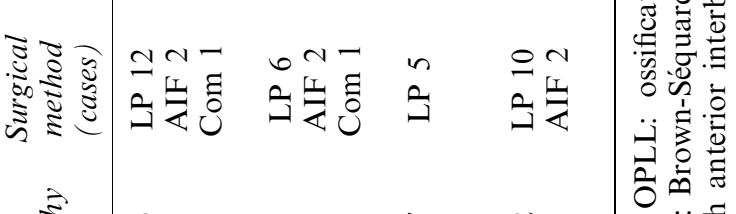

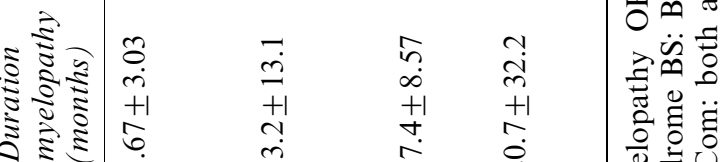

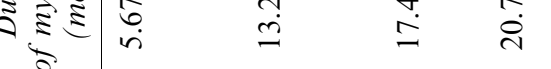

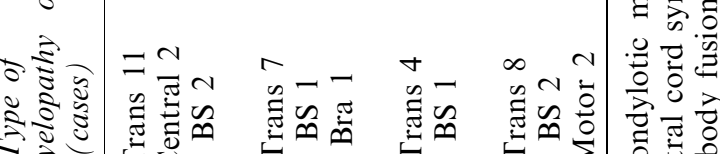

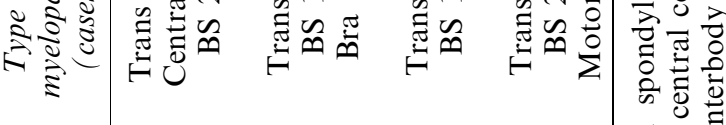

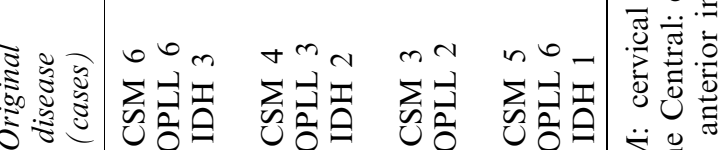

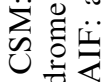

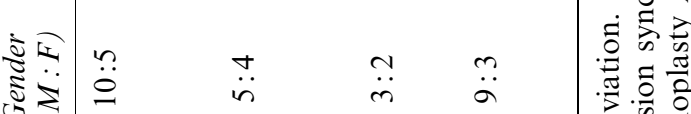

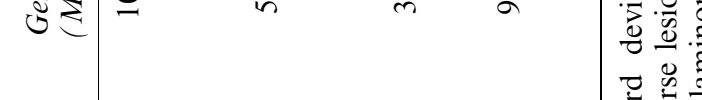

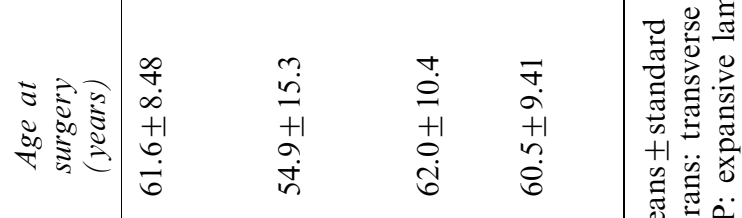


Table 3 Correlation between the recovery rate of JOA score and other clinical parameters

\begin{tabular}{lll}
\hline High intensity region in T2-weighted MRI & & $\begin{array}{l}P=0.0811 \\
P=0.582\end{array}$ \\
Gender & & $P=0.406 \quad$ Mann-Whitney's U test \\
Surgical method & & $P=0.483$ \\
Type of myelopathy & & $P=0.922$ \\
Original disease & $r=0.641$ & $P<0.0001 \quad$ Kruskal-Wallis rank test+ \\
HBO effect* & $r=0.381$ & $P=0.0184$ \\
Grip-and-release test* & $r=0.334$ & $P=0.110$ \\
Transverse area in CTM & $r=-0.126$ & $P=0.388$ \\
Duration of myelopathy & $r=0.190$ & $P=0.236$ \\
Spinal canal diameter & $r=0.149$ & $P=0.347$ \\
Preoperative JOA score & $r=-0.130$ & $P=0.397$ \\
Age at surgery & $r=-0.113$ & $P=0.503$ \\
Compression ratio in myelography & & Spearman's rank correlation \\
\hline
\end{tabular}

*There was a statistically significant correlation.

Table 4 Correlation between the HBO effect and other clinical parameters

\begin{tabular}{|c|c|c|c|}
\hline $\begin{array}{l}\text { High intensity region in T2-weighted MRI* } \\
\text { Gender }\end{array}$ & & $\begin{array}{l}P=0.0435 \\
P=0.698\end{array}$ & Mann-Whitney's U test \\
\hline $\begin{array}{l}\text { Type of myelopathy } \\
\text { Original disease } \\
\text { Surgical method }\end{array}$ & & $\begin{array}{l}P=0.289 \\
P=0.586 \\
P=0.675\end{array}$ & $\begin{array}{l}\text { Kruskal-Wallis rank test }+ \\
\text { Fisher's PLSD }\end{array}$ \\
\hline $\begin{array}{l}\text { Grip-and-release test } \\
\text { Transverse area in CTM } \\
\text { Duration of myelopathy } \\
\text { Spinal canal diameter } \\
\text { Preoperative JOA score } \\
\text { Age at surgery } \\
\text { Compression ratio in myelography }\end{array}$ & $\begin{aligned} r & =0.307 \\
r & =0.267 \\
r & =-0.150 \\
r & =0.110 \\
r & =0.084 \\
r & =-0.079 \\
r & =-0.019\end{aligned}$ & $\begin{array}{l}P=0.089 \\
P=0.310 \\
P=0.184 \\
P=0.257 \\
P=0.809 \\
P=0.814 \\
P=0.668\end{array}$ & Spearman's rank correlation \\
\hline
\end{tabular}

*There was a statistically significant correlation.

$+0.573 \times$ the grip-and-release test (counts $/ 10 \mathrm{~s})$ (multiple regression coefficient $=0.753, P<0.0001)$.

\section{Discussion}

Various attempts have been made to predict the surgical prognosis of compression myelopathy. Kifune $^{10}$ and Mimatsu et $a l^{13}$ performed multivariate analysis using clinical parameters such as age at surgery, disease duration, and preoperative severity of myelopathy. They concluded that these clinical parameters could predict the surgical prognosis with multiple regression coefficients ranging from 0.68 to 0.727 . Fujiwara et $a l^{2,3}$ have reported that the transverse area of the spinal cord at the most severe site of compression measured using CTM efficiently reflects the pathological changes of the spinal cord and surgical prognosis. He also stated that the correlation coefficient between this parameter and the recovery rate of JOA score after surgery is 0.549 , and that a transverse area of the spinal cord of $30 \mathrm{~mm}^{2}$ represents a critical point at which irreversible changes occur in the spinal cord. Koyanagi et $a l^{11}$ have reported that it is possible to predict the surgical prognosis to a multiple regression coefficient of 0.74 with multiple regression analysis using the transverse area of the spinal cord at the most severe site of compression and disease duration.

Recently, an intramedullary high intensity region in the spinal cord on T2-weighted saggital MRI has attracted attention, and it has been reported that there are many cases with an unfavorable outcome in the group with such intramedullary changes. ${ }^{12,16}$ In contrast, another report described a lack of association between such changes and the surgical outcome. $^{7,20}$ Thus, no consensus has been reached regarding the significance of such enhancement in the spinal cord on T2-weighted MRI.

The effect of $\mathrm{HBO}$ showed a high correlation with the recovery rate of JOA score after surgery as compared to other hitherto investigated parameters, and multiple regression analysis using the effect of $\mathrm{HBO}$ and the grip-and-release test facilitated prediction of a truly precise recovery rate of JOA score after surgery (multiple regression coefficient 0.753). In particular, in the group in which there was preoperatively no response to $\mathrm{HBO}$, the mean recovery rate was only $30 \%$. The rate was significantly worse than 
that of the groups which responded to $\mathrm{HBO}$, suggesting the existence of irreversible changes of the spinal cord.

The fact that symptoms were transiently improved by $\mathrm{HBO}$ suggests that the local hypoxia in conjunction with mechanical compression is one of the causes of compression myelopathy. Kelly et al ${ }^{9}$ in canine experiments have demonstrated that $\mathrm{HBO}$ improves the hypoxia accompanying spinal cord injury and enhances functional recovery of the injured cord. Ono et $a l^{14}$ have identified an inhomogenious distribution of degeneration foci and extensive cavity formation of the gray matter as characteristic pathological findings of compression myelopathy, and have implicated obliteration of the intrinsic vessels of the gray matter. In addition, Yoshizawa et $a l^{23}$ have demonstrated the importance of obliteration of the subarachnoid space resulting in interference with the oxygen supply derived from the cerebrospinal fluid.

Regarding the optimal pressure of $\mathrm{HBO}, 2.5$ atmospheres has been recommended from the viewpoint of the diffusion of oxygen to the ischemic spinal cord as well as the prevention of oxygen toxicity. ${ }^{18}$ When the pressure is elevated to 2.5 atmospheres under $100 \%$ oxygen, the arterial oxygen tension at $1900 \mathrm{mmHg}$ theoretically reaches approximately 20fold the physiological value and enhances the diffusion of oxygen to the tissues.

Although numerous reports describing the application of $\mathrm{HBO}$ in the treatment of spinal cord injury and compression myelopathy are available, in almost all cases it was used as adjuvant therapy in operated cases. $8,19,21,22$ In the present study as well, symptomatic improvement was limited to a slight amelioration of subjective symptoms such as numbness or clumsiness of the fingers, and this effect was only temporary in most cases. These results emphasize the limitations of $\mathrm{HBO}$ as a therapeutic approach.

In conclusion, $\mathrm{HBO}$ is a useful diagnostic tool to predict the surgical outcome in cervical compression myelopathy.

\section{References}

1 Crandall PH, Batzdorf U. Cervical spondylotic myelopathy. $J$ Neurosurg 1966; 25: 57-66.

2 Fujiwara K et al. Morphometry of the cervical spinal cord and its relation to pathology in cases with compression myelopathy. Spine 1988; 13: $1212-1216$
3 Fujiwara $\mathrm{K}$ et al. The prognosis of surgery for cervical compression myelopathy. An analysis of the factors involved. $J$ Bone Joint Surg [Br] 1989; 71-B: 393-398.

4 Gamache FW, Myers RA, Ducker TB, Cowley RA. The clinical application of hyperbaric oxygen therapy in spinal cord injury: A preliminary report. Surg Neurol 1981; 15: 85-87.

5 Hirabayashi $\mathrm{K}$ et al. Expansive open-door laminoplasty for cervical spinal stenotic myelopathy. Spine 1983; 8: 693-699.

6 Itoh T, Tsuji H. Technical improvements and results of laminoplasty for compressive myelopathy in the cervical spine. Spine 1985; 10: $729-736$.

7 Itoh Y et al. Preoperative and postoperative evaluation of clinical symptoms of cervical myelopathy, with special reference to T2 value of MRI. Rinsho Seikei Geka 1993; 28: 1221-1227.

8 Kawakami K et al. Hyperbaric oxygen therapy on cervical cord injury following emergency surgery. Spine Spinal Cord 1994; 7: $399-403$.

9 Kelly DL, Lassiter KR, Vongsvivut A, Smith JM. Effects of hyperbaric oxygenation and tissue oxygen studies in experimental paraplegia. J Neurosurg 1972; 36: 425-429.

10 Kifune M. Prediction of the postoperative results of cervical spondylotic myelopathy. A study by multivariate analysis. Cent Jpn J Orthop Traumatol 1989; 32: 2012-2021.

11 Koyanagi T et al. Prediction of the recovery rate after surgery for cervical myelopathy from the view of CT-myelography. Rinsho Sekei Geka 1991; 26: 3-10.

12 Matsuda Y et al. Increased MR signal intensity due to cervical myelopathy. Analysis of 29 surgical cases. J Neurosurg 1991; 74: $887-892$.

13 Mimatsu K et al. Prediction of prognosis of cervical myelopathy. Seikeigeka 1991; 42: 1576-1582.

14 Ono K, Ohta H, Tada K, Yamamoto T. Cervical myelopathy secondary to multiple spondylotic protrusions. A clinicopathologic study. Spine 1977; 2: 109-125.

15 Ono K et al. Myelopathy hand. New clinical signs of cervical cord damage. J Bone Joint Surg [Br] 1987; 69-B: 215-219.

16 Sato $\mathrm{T}$ et al. Intramedullary enhanced region in MRI of cervical spondylotic myelopathy. J Orthop Trauma Surg 1993; 36: $917-$ 922.

17 Simmons EH, Bhalla SK. Anterior cervical discectomy and fusion: a clinical and biomechanical study with eight-year followup. J Bone Joint Surg [Br] 1969; 51-B: 225-237.

18 Shin RW, Yagi H, Kusuda K, Koyama M. Efficacy of hyperbaric oxygenation therapy for acute or chronic spinal cord regions. Jpn J Hyperbaric Med 1990; 25: 113-117.

19 Tatsumura $\mathrm{T}$ et al. Clinical significance of hyperbaric oxygen therapy in the treatment of cerebro-spinal diseases. Jpn $J$ Hyperbaric Med 1990; 25: $169-176$

20 Wada E, Ohmura M, Yonenobu K. Intramedullary changes of the spinal cord in cervical spondylotic myelopathy. Spine 1995; 20: $2226-2232$

21 Yeo JD, Lowry C, Mckenzie B. Preliminary report on ten patients with spinal cord injuries treated with hyperbaric oxygen. Med $J$ Aust 1978; 2: $572-573$.

22 Yoshida T et al. Clinical evaluation of the hyperbaric oxygen therapy for treatment of the spinal cord compression myelopathy. Jpn J Hyperbaric Med 1988; 23: 109-115.

23 Yoshizawa H. Blood supply of spinal cord and cerebrospinal fluid flow. Nichidoku-iho 1995; 40: $84-95$. 